\title{
The Structure of Retrospective Accounts of Family Environments: Related to the Structure of Personality Attributes
}

\author{
Gerard Saucier \\ Department of Psychology \\ University of Oregon \\ Kelly R. Wilson \\ Department of Psychology \\ Stanford University \\ Jette Warka \\ Department of Psychology \\ Loma Linda University
}

\begin{abstract}
Environmental variables are often assumed to constitute a qualitatively different domain from personality variables, and unlike personality variables, their structure is relatively poorly understood. We studied retrospective descriptions of families-of-origin in 3 samples using the items of Moos's (1974) Family Environment Scale (FES). Using cluster-analytic procedures, we generated a new set of 22 homogeneous item clusters for the FES at a level more specific than the 10 conventional FES scales, thus enhancing potential fidelity in the measurement of family environments. We show that these item composites are well organized under a 3-factor structure and that reports of family environments on these 3 factors correlate moderately with those of the Big Five personality factors that have the most analogous content. Results suggest possible homology between the structure of family environments and the structure of personality dispositions.
\end{abstract}

Across the various domains of psychology, researchers have tended to separate variables related to persons from variables related to environments. One origin of this separation may be Lewin's (1936) classic dictum that behavior is a function of person and environment. However, Lewin's gestalt approach to understanding social phenomena led him to be an advocate of finding methods that could represent the person and the environment in common terms, enabling psychologists to describe and study the whole situation. Although Lewin's suggestion to develop common lexical representations for both person and environment has been echoed by others (e.g., Frederiksen, 1972; Sells, 1963), the structure of attributes of environments has been given far less attention than that of persons. Certainly, laboratory environments have been created by experimental psychologists who are eager to learn what effect the "situation" has on the person, but these manipulations have not advanced the creation of a suitable taxonomy of environmental variables (Frederiksen, 1972).
Hence, person and environmental variables have historically (and necessarily) been handled by separate methodological approaches.

The distinction between person variables and environmental variables is often too rigid. Both represent predicatesthings that can be said about a person. Consider the statements "Robin is tall" and "Robin comes from a very close family." Here, "is tall" and "comes from a very close family" are both predicated of Robin. One describes a trait and the other describes an environment, but both describe a person. Accordingly, one can apply those statistical methodologies used to study trait structure to study the structure of environmental descriptors.

The relation and possible overlap between structures of traits and structures of environments are of obvious interest. Psychodynamic theory and current empirical research (Pincus \& Ruiz, 1997) suggests that assessing one's early interpersonal environment is essential in understanding adult 
personality processes and later psychopathology. Environmental variables also influence the phenotypic expression of behavior. It is often thought that the cultural and social characteristics of the environment are reflected in individual behaviors and personality trait manifestations (Magnusson, 1981a; McCrae et al., 2000). In addition, individuals differ in the ways in which they perceive and construe the environment, and hence, environment perception is potentially a meaningful form of individual differences (Magnusson, 1981b), part of the individual's mind-set if not his or her personality.

Previous research has shown a robust relationship between the family environment-often assessed through scales that measure parental behaviors (Plomin, McClearn, Pedersen, Nesselroade, \& Bergeman, 1988) or parental representations-and personality traits. For example, individuals who report memories of parental figures as loving possess lower levels of neuroticism and higher levels of extraversion, agreeableness, conscientiousness (Pincus \& Ruiz, 1997) and openness to experience (McCrae \& Costa, 1988). McCrae and Costa (1988) also found that having had casual (vs. demanding) parents was associated with lower Conscientiousness scores in adulthood and with low Extraversion and high Openness.

In addition, studies that have examined the structure of parental representations have tended to characterize them as yielding a small number of meaningful factors. Rumpold, Doering, Hofer, and Schussler (2002) factor analyzed a measure of parental style and found factors interpreted as Indifference, Abuse, and Over-control; the latter two of these factors seem to correspond to Calm-Conflictual and Structuredness. In an analysis of spontaneous descriptions of parents, Quinlan, Blatt, Chevron, and Wein (1992) found three distinct conceptual factors: Benevolent, Punitive, and Ambitious. The same spontaneous description task has also been found to yield three alternative factors, best described as Agency, Communion, and Structure (Heck \& Pincus, 2001). The Family Environment Scale (FES; Moos, 1974) was constructed to measure the social and necessarily interpersonal climates of families. Therefore, it is not surprising that the structure of parental representations will, at the very least, fit under the umbrella structure of family environments, if not yield the same pattern. One should expect the structures of self-representations of personality traits, of parental representations, and of family environments-all descriptors of behavioral tendencies of people-to resemble each other because they might all be considered common expressions of a mind-set, a partially consistent set of beliefs and expectancies about people, in the respondent.

An individual encounters a range of environments in the course of even 1 day including work (or school) and home environments and environments populated with friends, or family, or neither (Saucier, Bel-Bahar, \& Fernandez, in press). In this article, we examine the structure of one type of environmental descriptors-retrospective accounts of family-oforigin environments operationalized in the items of an FES.
Then, we compare this structure to structures derived from studies of personality traits.

Following Frederiksen (1972), we have taken advantage of an existing classification that describes a subtype of environments, namely, the family environment, in the hopes of illuminating the structure of these descriptors. We note that the family environment is related to some other environments, such as groups of friends and peers, in that it comprises individuals that influence and are influenced by one another, thus serving as cocreators of a shared environment. In contrast to friends and peer groups, however, the family can be considered of special status in that the interactions family members have with one another are typically more intense than those they have with people outside the family (Beavers, 1977, p. 27) and also tend to be more enduring across time.

\section{ACCOUNTS OF THE STRUCTURE OF FAMILY ENVIRONMENT VARIABLES}

How can family environment be assessed? Moos (1974; Moos \& Moos, 1981, 1986) has created an instrument that devised family-environment items based on information gathered from observations and interviews with families as well as a theoretical conception of three ways in which one might differentiate among families_-personal growth, quality of interpersonal relationships, and system maintenance (Moos, 1990). Content and face validity were built into the FES subscales by defining constructs and then "preparing items to fit the construct definitions, and selecting items that were conceptually related to a dimension" (Moos, 1990, p. 200). Thus, the scale-construction approach was rational or intuitive (Goldberg, 1972). It involved initially 12 hypothesized dimensions and a reduction from 200 initial to 90 final items. A diverse sample ( $N>1,000$ from 285 families) was used as an empirical derivation sample. Items were selected based on (a) lack of skew in the response distribution, (b) ability to discriminate between families, (c) positive correlation with other items on their subscale, and (d) high correlation with their targeted subscale relative to other subscales.

In the final version of the FES, the items were sorted into 10 subscales, each consisting of nine items. The conceptual area of Personal Growth consists of the Independence, Achievement Orientation, Intellectual-Cultural Orientation, Moral-Religious Emphasis, and Active-Recreational Orientation subscales; the Quality of Relationships area consists of the Cohesion, Expressiveness, and Conflict subscales; and the System Maintenance area consists of the Organization and Control subscales.

Moos (1990) reported that 12-month subscale stabilities for the FES ranged from .59 to .67, and 48-month subscales stabilities ranged from .45 to .54 . Internal consistencies for FES subscales reported in the manual ranged from .64 to .79 (Moos, 1974). Moos (1990) considered these reliabilities acceptable. Roosa and Beals (1990), however, obtained lower coefficients (from .46 to .71) for five of the FES subscales 
in a sample that consisted primarily of well-educated, EuroAmerican women.

The FES subscale reliabilities might be improved by increasing the length of each subscale, which consists of only nine items. Another approach would be to break the subscale items into more homogeneous clusters. Internal consistency does not guarantee unidimensionality (Schmitt, 1996; Streiner, 2003). It is possible that there are multiple dimensions within one or more of the conventional FES subscales, and this multidimensionality may attenuate internal consistency and relations with external correlates. A careful examination of the literature surrounding the construction of the FES suggests that further study of the FES item pool is warranted. Alternate ways of aggregating the items may lead to more information (i.e., more than 10 subscales), an elucidation of the underlying constructs being measured, and/or higher reliabilities.

Studies of the factor structure of the FES have produced varying results. Moos (1990; Moos \& Moos, 1986) has reported that factor analyses of the FES subscales have yielded useful solutions of two to six factors and that item-level factor analyses have tended to find eight-factor solutions, although "the specific item composition of the eight factors has varied" (Moos, 1990, p. 206). Moos (1990) attributed the variability to a general rule that "the factor structure of an assessment procedure depends largely on the sample used in the analysis," noting that "more factors are likely to emerge in more heterogeneous samples" (p. 206). Additionally, FES items have a dichotomous (true-false) response format, and one should be cautious about item-level factor analysis with such items because they are more prone than multipoint items to yield spurious outcomes (Nunnally \& Bernstein, 1994). Therefore, we emphasize subscale-level factor analyses.

Fowler (1981) analyzed the matrix of subscale intercorrelations in the FES normative sample using maximum likelihood methods. A scree test suggested two factors. The varimax-rotated versions of these factors included one defined most strongly by the Cohesion subscale (and secondarily by Expressiveness, Independence, and the IntellectualCultural and Active-Recreational Orientation subscales, with the Conflict subscale having a negative loading); Fowler (1981) related it to relationship and personal-growth issues. The other factor was defined by the Control, Achievement Orientation, Moral-Religious Emphasis, and Organization subscales, and Fowler (1981) related this factor to issues concerning "the stability and integrity of the family unit" (p. 163). Later, Fowler (1982) replicated these "Interpersonal Cohesion" and "Control" factors in a smaller sample and found that partialing out a social desirability index (from the Personality Research Form, Form E; Jackson, 1974) affected the level of the subscale loadings but not the content of the factors. Fowler's $(1981,1982)$ two-factor solution is to date the best replicated structure of FES subscales; it has been replicated in two subsequent studies (Beavers, Boake \& Salmon, 1983; Oliver, Handal, Enos, \& May, 1988).
Other studies, however, have found three factors among the FES subscales. Oliver, May, and Handal (1988) analyzed two normative samples (one adult, one adolescent) from the FES manual (Moos \& Moos, 1981). Two of the three factors resembled Fowler's (1981, 1982) factors; a third factor, similar across both samples, was labeled "Activities", and "was characterized by moderate to high loadings of several subscales of the Personal Growth dimension (IntellectualCultural Orientation, Active-Recreational Orientation, and Independence) coupled with a moderately low loading of Cohesion" (Kronenberger \& Thompson, 1990, p. 381). Kronenberger and Thompson (1990) examined the generalizability of these factors in additional samples of chronically-ill and nonchronically-ill individuals. There was substantial crosssample replication as indicated by coefficients of congruence of .90 or better between structures from the two types of samples. In both cases, one of the three factors was defined by the Active-Recreational and Intellectual-Cultural Orientation subscales combined with Expressiveness and Independence subscales; Kronenberger and Thompson labeled this factor "Supportive." The other factors were labeled "Conflicted"with Conflict versus Cohesion subscales being defining the factor in both types of samples-and "Controlling," with Control, Achievement, and Moral-Religious Emphasis as defining subscales in both types of samples. The three-factor solution accounted for more subscale variance than the twofactor solution and provided high communality values for all FES subscales.

The appearance of three factors in the FES subscales is not completely surprising because Moos (1974) constructed these subscales with three dimensions in mind: interpersonal relationships, system maintenance, and personal growth. These three dimensions have conceptual similarity to, respectively, the Cohesion factor, the Control factor, and the Supportive or Activities factor obtained in later studies. However, Moos (1974) conceived of personal growth as being exemplified by the Achievement and Moral-Religious Emphasis subscales, whereas the corresponding factor in later studies represents a more humanistic notion of personal growthemphasizing activities, expressiveness, and independence.

\section{RELATING THE STRUCTURES OF FAMILY ENVIRONMENT AND OF PERSONALITY}

Interestingly, the three FES factors seem homologous with factors found in previous studies of attributes of individual persons. Although a five-factor structure (the Big Five or the Five-factor model; Goldberg, 1990; Costa \& McCrae, 1992) of personality attributes has become widely known, in fact, these five factors are derived mainly from studies of the lexicons of Germanic languages only (Saucier $\&$ Goldberg, 2001). Only three of these factors have been more widely replicated across European languages in studies of the personality descriptors that are found in lexicons (De Raad \& Peabody, 2005; Di Blas \& Forzi, 1999; 
Saucier, 1997; Saucier, Ostendorf, \& Peabody, 2001). These three personality factors-Extraversion, Agreeableness, and Conscientiousness - are sometimes called the "Big Three."

These Big Three have some conceptual similarity to the three factors derived from FES subscales. The Extraversion and Supportive factors both reference an active, expressive orientation to the world outside (whether outside the individual or outside the group) involving agency and independence on the part of actors. The Agreeableness and Cohesion factors both contrast helpful behaviors with angry and aggressive behaviors and tend to link the cohesiveness of relationships to friendly interactions with others. The Conscientiousness and Control factors both emphasize rule-oriented behavior whether it be in the form of individual orderliness, punctuality, dependability, consistency, and industriousness or in the form of set rules and procedures in family life. The other two Big Five factors are less behavioral in reference-Emotional Stability (or Neuroticism) referring to affective attributes and Intellect (or Openness) referring to cognitive attributes - and thus may generalize less well to the attributes of environments in which groups of people function and where affective and cognitive elements may decrease in importance relative to behavioral ones. Saucier (1997) found these latter two factors to be less replicable in peer-rating data than in self-rating data unlike the Big Three that are similarly replicable in both types of data.

Among the existing FES subscales, some reflect attributes that are used to describe persons as well as families (Independence, Organization, Achievement Orientation), and most of the FES constructs could be adapted to describe individuals. For example, Expression might reflect individual dispositions toward expressiveness, Conflict might reflect conflict proneness, Moral-Religious Emphasis might reflect individual morality and religiousness, Control might reflect one's tendency to control others, and Intellectual/Cultural Orientation and Active-Recreational Orientation could be adapted to readily differentiate individuals as well as families.

There are, however, few studies that have examined correlations between FES scores and scores on personality measures. Sines (1984) examined correlations between FES subscales and Minnesota Multiphasic Personality Inventory (MMPI; Hathaway \& McKinley, 1943) Clinical Scales. The highest correlations (averaging .26-.31 in magnitude across male and female subsamples) were between the MMPI Pd (Psychopathic Deviate) scale and the Conflict (.30) and Cohesion (-.31) subscales and between the MMPI F (Infrequency) scale and the Conflict (.26), Cohesion (-.26), and Achievement (-.30) subscales. These relations suggest, unsurprisingly, that deviance (whether behaviorally or in response style) tends to be associated with reports of family environments that were high in conflict but low in cohesion and achievement orientation. Another significant correlation was found between the MMPI Si (Social Introversion) and the Expressiveness subscale $(-.26)$, relations that suggest personality-environment congruence: Relatively extraverted individuals describe their family interactions as having somewhat higher than average extraverted characteristics.

A study (Forman \& Forman, 1981) that examined the relation between FES scores and adolescents' responses to Cattell's (1958) High School Personality Questionnaire concluded that FES Expressiveness correlated with adolescent sociability. Forman and Forman (1981) also found a high degree of concordance between FES Independence and adolescents appearing outgoing, independent, and relaxed and between FES Achievement Orientation and adolescents' self-reported enthusiasm, independence and self-sufficiency. These results are likewise consistent with a tendency for the attributes of family environments to be somewhat similar to the individual attributes of the persons found within them.

Why would one expect relations between personality and family environment variables? If such correspondences exist in objectively observable phenotypes and not just schematized perceptions, the correspondences could be due to the effects of family environment on the child's phenotypic characteristics or to sharing of genetic dispositions among family members. Another account would relate the correspondence not to actual overlap between individual and environmental attributes but instead to schemas. Individuals might have a tendency toward activating "three-factor schemas" when completing these types of measures. These schemas may be a common source for the Big Three of personality and the three FES factors.

Moreover, these schemas may also be partially responsible for some other, related three-factor structures. Osgood, Suci, and Tannenbaum (1957) proposed, based on studies of people's perceptions of a wide variety of objects and only or even mainly perceptions of people, that the three dimensions of Activity, Evaluation, and Potency are universal features of meaning in natural language. Osgood (1966) found three closely related dimensions in facial expressions-Activation, Pleasantness, and Control-that seem conceptually similar to both the FES factors and the related factors of personality attributes. Mulaik (1964) was perhaps the first psychologist to notice possible relations between the structures of personality attributes and the three dimensions of Evaluation, Activation, and Potency. Mulaik's (1964) view was that "anyone who has worked with factor-analyzing trait scales and who also has a familiarity with Osgood's work will be struck by the similarity between his factors of meaning and the factors typically found for trait ratings" (p. 507). Osgood (1969) saw these three semantic dimensions as resting on "the innateness of the emotional reaction system of the human animal" (p. 195). These dimensions have therefore been described as the affective-meaning system and/or the system by which humans attribute emotions or feelings to the persons and things in their environment (Osgood, 1974). This affective-meaning system - a set of relatively ubiquitous schemas - has been hypothesized to be the basis on which the invariance of the structure of personality ratings rests (Kuusinen, 1969; Osgood et al., 1957). The three 
Osgood (1969) dimensions do not, however, correspond neatly to the Big Three described previously.

If there is commonality between the structures of personality and of environments, there are two important questions. Is the commonality more highly related to Osgood's (1969) dimensions or to a phenotypic Big Three structure? Also, theoretically related, does the commonality stem from generic schemas (e.g., an affective-meaning system), or from the structure of external reality (i.e., of real phenotypes), or some combination of the two?

If, on the other hand, there is little commonality between structures of traits and environments or little empirical relation between the characteristics of an individual's traits and his/her environment, a competing view becomes more plausible. In this interactional view, humans are not entities in and of themselves; rather, individuals interact with others and in situations. These interactions often result in predictable situation-specific behaviors that are atypical for one or more of the actors outside of the given context. For example, individuals who are not especially hostile when they are alone can act with tremendous anger when confronted with a hostile other or an anger-inducing situation. To the extent to which this account is true, relations between family environments and stable dispositions should be minimal, and this account might be used as an explanation if such relations involve small effects.

\section{HYPOTHESES}

The best replicated structure for FES subscales has been one with two factors (Fowler, 1981, 1982), so it is worthwhile to check its replication in new samples. However, for this instrument, three-factor structures seem more theoretically interesting. We hypothesized that a three-factor structure for the FES would be found (a) in our samples when using the existing 10 FES subscales and (b) when items are aggregated alternatively into new content clusters that have more reliability. We also hypothesized that respondents' scores on these three factors of the FES would correlate (c) with their respective scores on the Big Three personality factors, that is, for Extraversion, Agreeableness, and Conscientiousness. A competing hypothesis was that the FES factors would correlate (d) with scores on adjectives representing Osgood et al.'s (1957) affective-meaning factors. Hypotheses (c) and (d) can be understood as competing hypotheses. Our study examines the viability of all four hypotheses.

\section{METHOD}

\section{Participants}

We made use of three samples of participants. The first two fit our purposes because they involved collection of personality ratings as well as the FES. Samples 1 and 2 included individuals recruited for a study on the biological bases of alcoholism and drug addiction, with Sample 1 being recruited as a "case" sample and Sample 2 as a "control" sample. Sample 1 consisted of 212 participants ( 208 men, 4 women) in an addictions treatment program at a U.S. Veterans Administration medical center in California with a mean age of 41.3 years $(S D=7.3)$; ethnic background was $66 \%$ EuroAmerican, 19\% African American, and 15\% Hispanic. Sample 2 consisted of 252 students (143 men and 109 women) at a state university in California with a mean age of 33.6 ( $S D=7.8$ ); this sample was recruited so as to oversample older students, many of whom held full-time jobs concurrent with their program of studies. Ethnic background was 79\% Euro-American, 8\% African American, and 13\% Hispanic. Sample 3 consisted of 428 students (91 men and 337 women) from the same state university campus but included a greater number of young individuals; the mean age was $26.0(S D=$ 9.4); ethnic background information was available for almost $97 \%$ of respondents among whom 59\% were Euro-American, 19\% Hispanic, 10\% African American, 10\% Asian or Asian American, and $2 \%$ other. In total, we analyzed the responses of 892 persons in this study.

\section{Family-Environment Measure}

All participants completed the FES (Moos \& Moos, 1986), which consists of 90 true-false items, in reference to their family of origin. These items are scored on 10 subscales, with 9 items being scored on each subscale.

\section{Development of FES Item Clusters}

Because alternate ways of aggregating FES items may prove more informative, we developed a set of content-based item clusters as an alternative to the 10 conventional FES subscales. We derived clusters in Samples 1 and 2 only to preserve Sample 3 as a sample for cross-validating the internal consistency and factor analyses. Within each of these samples, we generated cluster-analytic dendrograms using correlations between items as the distance measure according to commonly used methods of average linkage between groups, average linkage within groups, and complete linkage (i.e., furthest neighbor). To assure that negatively correlated items could cluster together, we reverse scored each of the 90 items, and we conducted the cluster analyses on 180 variables (90 original-scored and 90 reverse-scored items). We identified sets of items that tended to cluster together across methods and across samples; these formed our initial item clusters. We then refined these initial clusters using criteria of (a) maximizing reliability for the cluster and (b) splitting clusters appearing to have two kinds of clearly distinguishable content.

Table 1 reports on the 22 item-clusters, including the item numbers of items included in each cluster, and their internal consistency (coefficient alpha) estimates in each of the three samples. Two example items from each cluster are listed in 
the Appendix. We labeled each cluster to reflect its unique content. Internal consistency coefficients ranged from .46 to .81 in the derivation samples $(M=.66)$ and from .42 to .82 in the cross-validation sample (Sample 3; $M=.63$ ). Scoring these 22 item-clusters requires only 72 of the 90 FES items; the other 18 FES items had relatively low correlations with all other items and did not cluster well with other items. For the most part, these item clusters function to divide each of the FES subscales into multiple finer grained subcomponents. However, some of the item clusters contain items from more than 1 of the 10 conventional FES subscales.

Table 2 presents the internal consistency estimates for these 10 FES subscales, which require for scoring administration of all 90 FES items. Is internal consistency higher in these longer scales? The mean alpha coefficient in the 10 subscales was .70 as compared with .65 for the 22 itemclusters. The difference was not large, which comes as a surprise because each of the 10 nine-item subscales is on average nearly three times longer than the clusters (which average only 3.2 items each). The internal consistency of the FES subscales was uneven. Although most of the subscales had higher reliability than the shorter clusters that utilize items from them, this was not always true for the Achieve-

\section{TABLE 1}

\section{Item Clusters From the Family Environment Scale}

\begin{tabular}{|c|c|c|c|c|}
\hline Cluster Label & Item Nos. & Sample 1 & Sample 2 & Sample 3 \\
\hline Solidarity & $31,61 \mathrm{r}$ & .70 & .80 & .75 \\
\hline Supportiveness & $1,51,81$ & .68 & .75 & .68 \\
\hline Open Disclosure & $2 \mathrm{r}, 32,54 \mathrm{r}, 62$ & .65 & .66 & .64 \\
\hline Upset Proneness & 22,52 & .55 & .65 & .60 \\
\hline Cool Temperedness & 13,33 & .75 & .73 & .57 \\
\hline Combativeness & $3,63 \mathrm{r}, 71 \mathrm{r}$ & .65 & .69 & .61 \\
\hline Violent Force & 23,53 & .63 & .68 & .62 \\
\hline Encouragement & $14,64,84 \mathrm{r}$ & .64 & .67 & .60 \\
\hline $\begin{array}{l}\text { Achievement } \\
\text { Orientation }\end{array}$ & $5,15,45,65 \mathrm{r}$ & .67 & .72 & .67 \\
\hline $\begin{array}{l}\text { Intragroup } \\
\text { Competitiveness }\end{array}$ & $25 r, 73,85$ & .46 & .46 & .50 \\
\hline $\begin{array}{l}\text { Education/Culture } \\
\text { Orientation }\end{array}$ & $16 r, 26,36 r, 66,86$ & .69 & .76 & .68 \\
\hline Dialogue/Discussions & $6,46 r, 82$ & .52 & .71 & .67 \\
\hline TV Watching & 76,87 & .60 & .72 & .53 \\
\hline $\begin{array}{l}\text { Recreational } \\
\text { Activities }\end{array}$ & $27 \mathrm{r}, 37,57 \mathrm{r}$ & .65 & .61 & .65 \\
\hline Hobby Orientation & 47,67 & .63 & .57 & .52 \\
\hline Religious Activities & $8,18 \mathrm{r}, 28,78$ & .76 & .81 & .82 \\
\hline $\begin{array}{l}\text { Fundamentalist } \\
\text { Beliefs }\end{array}$ & $38 \mathrm{r}, 58,88$ & .57 & .60 & .42 \\
\hline Neatness & $19,29 \mathrm{r}, 59,89$ & .73 & .68 & .70 \\
\hline Industriousness & $21,39,69,75$ & .64 & .58 & .57 \\
\hline Strictness & $20 \mathrm{r}, 40,48,50$ & .64 & .69 & .68 \\
\hline $\begin{array}{l}\text { Autocracy/ } \\
\text { Overbearingness }\end{array}$ & $10 \mathrm{r}, 30,43,44,60 \mathrm{r}$ & .71 & .68 & .72 \\
\hline Permissiveness & $12,34,42,70,90 \mathrm{r}$ & .55 & .65 & .60 \\
\hline$M$ & & .64 & .68 & .63 \\
\hline
\end{tabular}

Note. $N=212$ (Sample 1); $N=252$ (Sample 2); $N=428$ (Sample 3). $\mathrm{r}=$ item is reverse-scored. ment Orientation, Moral-Religious Emphasis, and Control subscales, and it was never true for the relatively incoherent Independence subscale. Our results tended to converge with those of Sanford, Bingham, and Zucker (1999) who found low internal consistency for the Expression, Independence, Achievement Orientation and Control subscales.

In comparing the 10 subscales and the 22 item-clusters, there was a trade-off between slightly higher reliability (on average) from the subscales and a large increase in informativeness from the item clusters. The 22 item-clusters yield a greater variety of information about family environments and thus much greater utility for researchers interested in familyenvironment variables. Although the clusters offer a large increase in informativeness and utility and sacrifice little in terms of reliability relative to the conventional subscales, in the remainder of this article, we present evidence using both ways of scoring FES items.

\section{Personality Measures}

All participants in Samples 1 and 2 completed the NEO FiveFactor Inventory (NEO-FFI; Costa \& McCrae, 1992), a 60item self-report instrument with five scales corresponding to Neuroticism, Extraversion, Openness to Experience, Agreeableness, and Conscientiousness. Participants in Sample 3 did not complete the NEO-FFI, as this was not part of the design for the study that led to their recruitment (Warka, 1996).

The Interpersonal Circumplex (Wiggins, Trapnell, \& Phillips, 1988) dimensions of Agency and Communion have

TABLE 2 Internal Consistency for 10 Conventional
Subscales of the Family Environment Scale
(FES)

\begin{tabular}{lcccc}
\hline & & \multicolumn{3}{c}{ Coefficient Alpha Reliability } \\
\cline { 3 - 5 } Cluster Label & Item Nos. & Sample 1 & Sample 2 & Sample 3 \\
\hline Cohesion & $1,11,21, \ldots, 81$ & .82 & .84 & .82 \\
Expression & $2,12,22, \ldots, 82$ & .51 & .71 & .70 \\
Conflict & $3,13,23, \ldots, 83$ & .82 & .82 & .78 \\
Independence & $4,14,24, \ldots, 84$ & .53 & .61 & .46 \\
Achievement & $5,15,25, \ldots, 85$ & .65 & .62 & .52 \\
$\quad$ Orientation & & & & \\
Intellectual- & $6,16,26, \ldots, 86$ & .76 & .80 & .75 \\
$\quad$ Cultural & & & & \\
$\quad$ Orientation & & & & \\
Active- & & & & \\
$\quad$ Recreational & $7,17,27, \ldots, 87$ & .51 & .75 & .72 \\
$\quad$ Orientation & & & & \\
Moral-Religious & $8,18,28, \ldots, 88$ & .71 & .77 & .74 \\
$\quad$ Emphasis & & & & \\
Organization & $9,19,29, \ldots, 89$ & .77 & .74 & .75 \\
Control & $10,20,30, \ldots, 90$ & .59 & .69 & .70 \\
$M$ & & .67 & .74 & .69 \\
\hline
\end{tabular}

Note. $N=212($ Sample 1$) ; N=252($ Sample 2$) ; N=428($ Sample 3$) . r=$ item is reversed scored. FES items are ordered such that every 10 th item is scored on the same subscale. 
been shown to be strongly related to two of the Big Five factors, namely, Extraversion and Agreeableness (McCrae \& Costa, 1989). Thus, Extraversion and Agreeableness scores from the NEO-FFI are a reasonable indicator for the axes of this circumplex.

Most of the participants in Sample 2 were instructed to describe themselves on 141 personality adjectives using a 9-point rating scale ranging from 1 (extremely accurate) to 9 (very accurate). We chose sets of these adjectives to yield measures of the three affective-meaning dimensions as applied in self-description. Osgood, May, and Miron (1975, Table 4:18) recommended various pancultural scales for a short-form semantic differential. These were bipolar pairings of adjectives (e.g., good-bad). We selected adjectives found in Osgood et al.'s (1975) table from the set of 141 terms to form indexes of Evaluation, Potency, and Activity. We did not have bipolar scales but instead aggregated sets of forward- and reverse-keyed items to yield a single score for each dimension. Adjectives indexing Evaluation were pleasant, agreeable, kind, friendly, desirable, and glad (forward keyed) versus unhappy, hostile, rough, and sad (reverse keyed). For Potency, the adjectives were powerful and deep versus weak and helpless. For Activity, the adjectives were noisy, emotional, exciting, warm, and cheerful versus cold and quiet. All adjectives were taken from Osgood et al.'s (1975) table except helpless, an obvious low-potency term added to raise the Potency scale to four items. Values of coefficient alpha for these scales were .82 (Evaluation), .63 (Potency), and .60 (Activity).

\section{Analyses}

We conducted exploratory factor analyses (principal factors, varimax rotations) on the 10 FES subscales separately in each of the three samples and then on the 22 item-clusters in each sample. Previous studies have specified the structure of the FES subscales with too little precision and replicability to provide the basis for a confirmatory factor analysis, and this is the first investigation of item clusters, so exploratory analysis is more appropriate. On an a priori basis, we gave special attention to the two- and three-factor solutions. We generated regression-based factor scores for each participant for each factor in each sample, and we correlated these with the scale scores for the NEO-FFI and the three affective meaning dimensions.

\section{RESULTS}

We extracted factors from the intercorrelations of the $10 \mathrm{FES}$ subscales in the three samples. In each sample, there were two eigenvalues greater than 1.00 and an elbow in the scree plot after two factors. Four- and five-factor solutions tended to yield some factors with either no salient variables or a single salient variable, and we disregarded them. For two- and three-factor solutions, the factors resembled those in previous studies overall, although not with perfect consistency for all subscales.

For the 22 item-clusters, all three samples yielded very similar scree plots. In Sample 3, for example, the first 10 eigenvalues were $6.81,2.66,1.64,1.23,1.07,0.87,0.78$, $0.76,0.72$, and 0.60 . In the four- and five-factor solutions, the fourth and fifth factors were anchored by inconsistent sets of variables across the three samples. In contrast, the solutions with two and three factors were highly consistent across samples. We concentrated on these solutions because of this cross-sample robustness. We began with a check on whether the two-factor solutions replicated those found in previous studies.

\section{Two-Factor Solutions}

For the conventional FES subscales, in all three samples, Cohesion, Expression, Active-Recreational Orientation, Independence, and (negatively) Conflict had their highest loadings on the first factor; this factor resembled Fowler's (1981) Interpersonal Cohesion. In all three samples, Organization and Achievement Orientation had their highest loadings on the second factor; this resembled Fowler's (1981) Control factor only to a moderate degree-the Control and MoralReligious Emphasis subscales did not consistently have their highest loadings on this factor.

We also examined the two-factor solutions generated by the 22 item-clusters with attention to whether they replicated the Fowler (1981) factors. In each sample, the first factor was defined (roughly in this order) by the Solidarity, Supportiveness, Encouragement, Dialogue/Discussions, Open Disclosure, Education-Culture Orientation, Recreational Activities, Cool Temperedness, and Hobby Orientation clusters as opposed to (roughly in this order) the Autocracy/Overbearingness, Combativeness, Upset Proneness, Violent Force, Intragroup Competitiveness, and TV Watching clusters. This factor definitely resembles Fowler's (1981) Interpersonal Cohesion factor. In each sample, the second factor was defined (roughly in this order) by the Strictness, Industriousness, Neatness, Achievement Orientation, and Fundamentalist Beliefs clusters; this factor resembled Fowler's (1981) Control factor. Fowler's (1981) two factors, thus, were better replicated among the 22 item-clusters than among the 10 conventional subscales.

\section{Three-Factor Solutions}

We next examined whether the three-factor solutions would resemble those that have been reported in previous studies. Table 3 provides factor loadings for the 10 FES subscales in each of the three samples. The factors tended to resemble the three-factor solutions of Kronenberger and Thompson (1990) in most respects. Like Kronenberger and Thompson's earlier study, Expression and Independence had their highest loading on one factor, and Intellectual-Cultural 
Orientation and Active-Recreational Orientation usually had their highest loading on this same factor; the factor emphasized personal growth themes. As in Kronenberger and Thompson's earlier study, Cohesion and Conflict had high loadings on opposing poles of a second factor, which emphasized quality of relationship interaction. Also like that earlier Kronenberger and Thompson study, Achievement Orientation and Moral-Religious Emphasis had high loadings on a third factor on which Organization and Control also had high loadings; this factor emphasized rules and goals. However, in Sample 3, the three factors were slightly rearranged: One factor opposed Expression and Control, whereas another associated Achievement Orientation and Moral-Religious Emphasis with Intellectual-Cultural Orientation and ActiveRecreational Orientation.

Table 4 presents the three-factor solutions for the 22 FES item clusters in each of the three samples. When using the 22 item-clusters instead of the 10 FES subscales, the

TABLE 3

Factor Loadings for 10 Conventional Subscales of the Family Environment Scale

\begin{tabular}{|c|c|c|c|c|c|c|c|c|c|}
\hline \multirow[b]{2}{*}{ Cluster Label } & \multicolumn{3}{|c|}{ Factor 1} & \multicolumn{3}{|c|}{ Factor 2} & \multicolumn{3}{|c|}{ Factor 3} \\
\hline & S1 & $S 2$ & S3 & $S 1$ & $S 2$ & $S 3$ & $S 1$ & $S 2$ & $S 3$ \\
\hline Expression & $.66^{a}$ & $.66^{a}$ & $.70^{a}$ & .20 & .22 & .11 & -.22 & -.04 & .17 \\
\hline Active-Recreational Orientation & $.55^{a}$ & $.74^{a}$ & .46 & .22 & .12 & .12 & .13 & .23 & $.51^{a}$ \\
\hline Intellectual-Cultural Orientation & $.66^{a}$ & $.64^{a}$ & .41 & .35 & .18 & .20 & .24 & .28 & $.61^{a}$ \\
\hline Independence & .35 & $.58^{a}$ & $.51^{a}$ & $.36^{a}$ & .15 & .16 & -.04 & -.18 & .03 \\
\hline Conflict & -.24 & -.37 & -.42 & $-.78^{a}$ & $-.68^{a}$ & $-.70^{a}$ & .11 & .07 & -.11 \\
\hline Cohesion & .58 & .57 & $.50^{a}$ & $.68^{a}$ & $.64^{a}$ & .47 & .16 & .23 & .47 \\
\hline Organization & .20 & -.03 & -.08 & $.57^{a}$ & $.46^{a}$ & $.58^{a}$ & .54 & .45 & .42 \\
\hline Achievement Orientation & .21 & .13 & -.15 & .00 & .03 & .07 & $.77^{a}$ & $.55^{a}$ & $.51^{a}$ \\
\hline Control & -.37 & -.58 & $-.78^{a}$ & -.05 & -.10 & .04 & $.69^{a}$ & $.72^{a}$ & .35 \\
\hline Moral-Religious Emphasis & .30 & .11 & .03 & $.36^{a}$ & .27 & .20 & .32 & $.33^{a}$ & $.41^{a}$ \\
\hline
\end{tabular}

$\mathrm{S}=$ sample; $\mathrm{S} 1 N=212 ; \mathrm{S} 2 N=252 ; \mathrm{S} 3 N=428$.

${ }^{a}$ Highest loading for variable.

TABLE 4

Factor Loadings for 22 Item Clusters from the Family Environment Scale

\begin{tabular}{|c|c|c|c|c|c|c|c|c|c|}
\hline \multirow[b]{2}{*}{ Cluster Label } & \multicolumn{3}{|c|}{ Active-Passive } & \multicolumn{3}{|c|}{ Calm-Conflictual } & \multicolumn{3}{|c|}{ Structuredness } \\
\hline & $S 1$ & $S 2$ & $S 3$ & $S 1$ & $S 2$ & $S 3$ & $S 1$ & $S 2$ & $S 3$ \\
\hline Education/Culture Orientation & $.70^{a}$ & $.70^{a}$ & $.68^{a}$ & .23 & .17 & .22 & .20 & .04 & .16 \\
\hline Recreational Orientation & $.63^{a}$ & $.53^{a}$ & $.58^{a}$ & .24 & .15 & .18 & .22 & .06 & .03 \\
\hline Hobby Orientation & $.61^{a}$ & $.63^{a}$ & $.58^{a}$ & .17 & .21 & .14 & .18 & -.05 & .12 \\
\hline Encouragement & $.58^{a}$ & $.60^{a}$ & $.57^{a}$ & .19 & .33 & .34 & .04 & -.07 & -.15 \\
\hline Open Disclosure & $.55^{a}$ & $.47^{a}$ & $.54^{a}$ & .26 & .33 & .36 & .09 & .07 & -.09 \\
\hline Dialogue/Discussions & $.54^{a}$ & $.74^{a}$ & $.62^{a}$ & .15 & .14 & .24 & .02 & .03 & -.04 \\
\hline Solidarity & $.54^{a}$ & .46 & $.59^{a}$ & .53 & $.50^{a}$ & .48 & .20 & .21 & .11 \\
\hline Religious Activities & $.41^{a}$ & $.34^{a}$ & $.44^{a}$ & .24 & .09 & -.04 & .26 & .31 & .25 \\
\hline TV Watching & $-.46^{a}$ & $-.45^{a}$ & $-.36^{a}$ & -.29 & -.22 & -.31 & -.12 & -.09 & -.19 \\
\hline Cool Temperedness & .00 & .11 & -.01 & $.73^{a}$ & $.63^{a}$ & $.62^{a}$ & .06 & .01 & .13 \\
\hline Supportiveness & .52 & .44 & .50 & $.53^{a}$ & $.62^{a}$ & $.53^{a}$ & .11 & .24 & .03 \\
\hline Autocracy/Overbearingness & -.41 & -.36 & -.32 & $-.71^{a}$ & $-.73^{a}$ & $-.72^{a}$ & .05 & .09 & .11 \\
\hline Combativeness & -.27 & -.29 & -.35 & $-.70^{a}$ & $-.74^{a}$ & $-.71^{a}$ & -.23 & -.14 & -.16 \\
\hline Violent Force & -.29 & -.17 & -.21 & $-.60^{a}$ & $-.58^{a}$ & $-.59^{a}$ & -.11 & -.12 & -.04 \\
\hline Intragroup Competitiveness & -.17 & -.07 & -.11 & $-.59^{a}$ & $-.53^{a}$ & $-.47^{a}$ & .22 & .11 & .10 \\
\hline Upset Proneness & -.29 & -.36 & -.26 & $-.58^{a}$ & $-.54^{a}$ & $-.60^{a}$ & .03 & .08 & .12 \\
\hline Strictness & .15 & -.04 & .09 & -.07 & -.11 & -.12 & $.76^{a}$ & $.75^{a}$ & $.74^{a}$ \\
\hline Industriousness & .32 & .12 & .26 & .10 & .03 & .16 & $.74^{a}$ & $.70^{a}$ & $.69^{a}$ \\
\hline Neatness & .17 & .05 & .10 & .25 & .21 & .29 & $.54^{a}$ & $.55^{a}$ & $.53^{a}$ \\
\hline Achievement Orientation & .48 & $.49^{a}$ & $.53^{a}$ & .05 & .13 & .16 & $.51^{a}$ & .41 & .33 \\
\hline Fundamentalist Beliefs & .21 & .07 & .19 & -.07 & .02 & -.07 & $.26^{a}$ & $.35^{a}$ & $.20^{a}$ \\
\hline Permissiveness & .13 & .29 & .14 & .01 & .26 & .18 & $-.67^{a}$ & $-.51^{a}$ & $-.66^{a}$ \\
\hline
\end{tabular}

$\mathrm{S}=$ sample; $\mathrm{S} 1 N=212 ; \mathrm{S} 2 N=252 ; \mathrm{S} 3 N=428$.

${ }^{a}$ Highest loading for variable. 
three-factor solutions were quite consistent across samplesmore consistent than that for the three-factor solutions that utilized the 10 conventional subscales. Of the 22 itemclusters, 20 had their highest loading on the same factor in all three samples compared to only four of the 10 subscales meeting this criterion.

Correlations between factors from these 22 item-clusters and those from the 10 subscales indicated high convergence in Samples 1 and 2. Best-match pairs of 22-cluster and 10subscale factors correlated .90, .87, and .86 in Sample 1; and $.86, .83$, and .80 in Sample 2. In Sample 3, the subscale factors involved a rotational variant so that each factor from the 22 item-clusters was correlated highly (correlations of .56-.77 in magnitude) with two of the factors from 10 subscales.

These three factors fit the Kronenberger and Thompson (1990) labels fairly well, but better labels can be devised. The first factor emphasized dynamic and active environments, whether the activities involve education, culture, recreation, hobbies, communication, and religion but not televisionwatching, a more passive activity whose cluster has a strong negative loading on the factor. This factor could be called Active Versus Passive. The second factor emphasized the extent to which coerciveness, conflict, and anger is present in the family environment: cool temperedness as opposed to autocracy, combativeness, violent force, competitiveness, and upset proneness. This factor could be called Calm Versus Conflictual. The first two factors appeared to correspond to the interpersonal axes of Agency and Communion (Wiggins, Trapnell, \& Phillips, 1988) but only by a loose criterion.

The third factor emphasized attention to rules and goals as contrasted with laxity. It might be called Strict Versus Permissive or Tight Versus Loose, although the strong association of industriousness and neatness with the strict/tight side suggest some other label may be better. Strictness, industriousness, and neatness (as well as achievement orientation) all share the theme of Structuredness, so that label is adopted here. Example items from the highest loading clusters on each factor are "Learning about new and different things is very important in our family" (high on Active-Passive), "Family members often criticize each other" (low on CalmConflictual), and "There is a strong emphasis on following rules in our family" (high on Structuredness).

Overall, the 22 item-clusters lead to a more consistent structure across samples than do the 10 conventional FES subscales. Thus, these clusters provide not only more information but also more structural consistency.

\section{Correlations With Personality}

We had predicted that scores on the three factors would correlate with respondents' scores on the Big Three personality factors: Extraversion, Agreeableness, and Conscientiousness. Table 5 provides correlations between the factor scores for the three-factor solution in Samples 1 and 2 based on FES item clusters and scores on the five NEOFFI broad-level scales. In line with predictions, Extraversion was correlated $(r=.33$ and $r=.39$ in Samples 1 and 2, respectively) with the Active-Passive factor, Agreeableness (.37 and .28) with the Calm-Conflictual factor, and Conscientiousness (.27 and .23) with the Structuredness factor. We had not predicted other correlations between FES factors and broad-level personality scales, but there were several important ones. Neuroticism was negatively correlated with both the Calm-Conflictual factor and the Active-Passive factor. Conscientiousness and Openness were positively correlated with the Active-Passive factor. Correlations between NEOFFI scales and factors from the 10 conventional FES subscales showed a similar pattern and similar magnitudes as did these factors from the 22 item-clusters.

The correlations of FES factors with Big Five factors beyond $\mathrm{E}$ and $\mathrm{A}$ undercut the view that a two-factor interpersonal circumplex will capture the most crucial dimensions in

TABLE 5

Correlations Between Factor Scores from 22 Item-Clusters and Self-Reported Personality Traits

\begin{tabular}{|c|c|c|c|c|c|c|}
\hline \multirow[b]{2}{*}{ Personality Score } & \multicolumn{2}{|c|}{ Active-Passive } & \multicolumn{2}{|c|}{ Calm-Conflictual } & \multicolumn{2}{|c|}{ Structuredness } \\
\hline & S1 & $S 2$ & S1 & $S 2$ & S1 & $S 2$ \\
\hline \multicolumn{7}{|l|}{ Five-Factor Domain Scales } \\
\hline Extraversion & $.33^{* * *}$ & $.39^{* *}$ & -.01 & .08 & .08 & $.14^{*}$ \\
\hline Agreeableness & .13 & .06 & $.37^{* * *}$ & $.28^{* * *}$ & -.01 & .09 \\
\hline Conscientiousness & $.35^{* * *}$ & $.15^{*}$ & .06 & .10 & $.27^{* * *}$ & $.23^{* * *}$ \\
\hline Neuroticism & $-.26^{* * *}$ & $-.22^{* * *}$ & $-.27^{* * *}$ & $-.28^{* * *}$ & -.01 & -.09 \\
\hline Openness to Experience & $.21^{* *}$ & $.12^{*}$ & -.13 & -.11 & -.01 & -.12 \\
\hline \multicolumn{7}{|c|}{ Affective-Meaning-Dimension Scales } \\
\hline Evaluation & & $.27^{* * *}$ & & $.30^{* * *}$ & & .12 \\
\hline Activation & & $.34^{* * *}$ & & .04 & & .11 \\
\hline Potency & & $.22^{* *}$ & & -.01 & & -.02 \\
\hline
\end{tabular}

$\mathrm{S}=$ sample.

${ }^{*} p<.05 .{ }^{* *} p<.01 .{ }^{* * *} p<.001$.

Note. S1 $N=211 ; \mathrm{S} 2 N=252$ for five-factor analyses and $N=216$ for affective-meaning-dimension analyses. 
family environments. It appeared that for the sake of comprehensiveness it would be necessary at least to add a third Structuredness factor to the two interpersonal axes (cf. Heck \& Pincus, 2001).

These correlations indicated considerable homology, generally a medium-sized effect, between a person's ratings of his or her own characteristics and similar characteristics ascribed to his or her family. Individuals who described themselves as relatively extraverted but also somewhat conscientious, open to experience, and low on neuroticism-a blend generally connoting an active rather than passive personality style-tended to describe their families as high on the Active-Passive dimension. Individuals who described themselves as relatively disagreeable and high on neuroticism-a blend characterized by hostility and antagonism - tended to describe their families as low in calmness and high in conflict, that is, they described families high in coercion, competition, and negative affect. Also, individuals who described themselves as relatively conscientious tended to describe their families as high on Structuredness-which emphasize rules, neatness, hard work, and nonpermissiveness.

If the family-environment factors corresponded neatly to Osgood's (1969) dimensions, which are thought to represent universal schemas, it would strengthen the argument that the homologies are due to common schemas underlying ratings both of personality and of family environment. The bottom half of Table 5 provides the correlations between the three FES-cluster factors and the scales representing affective-meaning dimensions. The Active-Passive factor was correlated positively with all three dimensional scales, whereas the Calm-Conflictual factor was correlated positively with only the Evaluation scale. Osgood et al. (1957) labeled the blend of Activation and Potency Dynamism and noted that this blend occurs commonly in judgments of about people and sociopolitical policies. Invoking this higher order blend, the FES-cluster Active-Passive factor might be considered positively valued Dynamism (Dynamism + Evaluation), whereas the Calm-Conflictual factor is related to Evaluation but independent of Dynamism. Most important, the Structuredness factor is unrelated to any affective-meaning dimension; this may indicate that the Osgood (YEAR) dimensions problematically lack content related to schemas for Structuredness. The lack of full correspondence between the three affective-meaning dimensions and the three FEScluster factors tends to undercut the notion that cognitiveaffective schemas of raters-schemas applied to ratings of any object or entity - are primarily responsible for the structure of family-environment correlations. It does not, however, rule out the notion that schemas related to the Big Three are related to this structure.

\section{DISCUSSION}

In this study, we demonstrated, first of all, the usefulness of a more informative way of scoring the FES by using 22 item-clusters instead of the 10 conventional subscales. Not only did 22 item-clusters provide more information than 10 subscales, but they appeared also to show more factorial consistency, especially with reference to a cross-validation sample separate from the samples in which the clusters were derived.

The study's structural hypotheses were confirmed. Thus, factors that have been found when analyzing FES subscales in previous studies were replicated in this study, although the 22 item-clusters reproduced these factors with more clarity and consistency than did the 10 subscales. In part, this may be due to the larger number of variables; factor analysis of data sets with a higher variable:factor ratio, as is true of the 22 item-clusters, have tended to produce more stable solutions (Guadagnoli \& Velicer, 1988; Widaman, 1990). The twofactor and three-factor solutions that has been found in this and previous studies have an easily understandable relation; both solutions had a Structuredness factor, but the threefactor solutions tended to yield divergent Active-Passive and Calm-Conflictual factors, whereas the two-factor solutions combined these into a single Interpersonal Cohesion (Active/ Calm versus Passive/Conflictual) factor that is broader and therefore seems more difficult to interpret.

\section{Correlations Between Personality and Family Environment Variables}

Previous studies have suggested that descriptions of family environments have a structure resembling that of descriptions of individual traits and particularly analogous to the first three factors (Big Three: Extraversion, Agreeableness, and Conscientiousness) of the Big Five. Our findings supported this view and more so than the view that family-environment factors correspond with the affective-meaning factors defined by Osgood (1969). With respect to the Big Three, not only were the structures somewhat similar, but individuals who scored high on a personality trait measure tended to score their family environment high on the analogous FES-cluster factor. A caveat is that the size of these correlations was moderate.

These findings should not be entirely surprising given previous literature has shown relations between the family environment and personality traits. For example, individuals who report memories of parental figures as loving possess lower levels of neuroticism and higher levels of extraversion, agreeableness, conscientiousness (Pincus \& Ruiz, 1997) and openness to experience (McCrae \& Costa, 1988). Consistent with findings of Pincus and Ruiz (1997), Table 5 indicates that favorable family environments - those high on both activeness and calmness-were associated with higher self-reports of a variety of favorable traits in adulthood. Consistent with findings of McCrae and Costa (1988) are the moderate correlations in Table 5 between Conscientousness and the Structuredness factor. However, inconsistent with McCrae and Costa (1988), Table 5 shows correlations with 
Structuredness of only .08 and .14 with Extraversion and -.01 and -.12 with Openness.

Of course, there are individual differences in raters' perceptions of the environment. Whereas many individuals are likely to rate attributes of their family environment as similar to their own personality attributes, others may be inclined to report dramatic discrepancies between their family's attributes and their own. These likely differences in perception deserve attention in future studies. However, it would be useful to compare individual personality traits with a more consensually validated account of a family environment such as might be obtained from the aggregated ratings of multiple family members.

One potential limitation, laid out by both Halverson (1988) and Plomin et al. (1988), is that retrospective reports of the entire family environment may perhaps tell us more about a single person than about the family in which he or she was raised. The typical individual seems to have a tendency to describe a family of origin that has attributes in common with his or her own personality. The seriousness of this limitation cannot be gauged, however, until more is known about the actual degree of valid similarity between an individual's traits and the traits of the environment in his or her family. To assess the degree of valid similarity, it is important to assess as much as possible actual rather than perceived characteristics of individuals and environments. Comparisons should be made between consensually validated ratings of both individual traits and family environments, in each case obtained from multiple well-informed raters and then aggregated into reliable composites. Longitudinal studies of this kind may shed light on how actual family environments and individuals' perceptions of them change over time.

\section{Possible Sources of Correlations Between Personality and Perceived Family Environment}

What are we to make of correlations, found in this study, between family environment variables and the personalities of the environments' inhabitants? These correlations may reflect the influence of a shared environment on personality dispositions, but they do not necessarily imply a direct environmental influence on personality. Adoption studies have shown that FES Personal Growth correlates positively with parent's ratings of their infant's Sociability and negatively with Emotionality (from the Colorado Childhood Temperament Inventory; Plomin \& DeFries, 1983) in nonadoptive homes but not in homes where the infant has been adopted (Plomin \& DeFries, 1983). Such findings suggest that some apparently environmental influences on personality could in fact be due to heredity by way of gene-environment correlation. Also, the attributes of family environments may be more a consequence than a cause of the attributes of the individuals in those environments. Correspondences may be due to genes or to environmental factors.
The correspondence may reflect a simple aggregation principle. We might conjecture that families having an environment with a high degree of independence probably contain relatively independent individuals, those with achievement orientation may contain relatively achievementoriented individuals, and so on. Thus, family environment attributes could be partly interwoven with, and even stem from, the attributes of the individuals in the family. Because the family system not only serves to shape individuals but is also defined by the members that comprise it, the attributes of a family environment could represent the mean of the individual attributes of its members: Hostile, conflict-prone individuals would foster hostile, conflictual family environments and vice versa. In this conception, akin to that which has been found in distributive models of culture (Goodenough, 1981; Schwartz, 1978), group-climate attributes are a function of the aggregated attributes of the group members. This aggregation model suggests that group attributes are a simple aggregate of constituent individual attributes (and as individual attributes change, those of groups may tend to also). These shared environment attributes that overlap with shared individual dispositions could, of course, be due to either common genetic or common environmental influences. The environmental influences would usually involve communication between family members and would be shaped not only by personality but also by the macrostructures of family ideology, shared religious values, geographical location, and cultural context.

Studies of behavior genetics have not been inconsistent with the aggregation model. Plomin, Chipuer, and Loehlin (1990; see also Plomin, 1994, chapter 4) conclude that "the factor structure of genetic correlations among traits and the structure of environmental correlations are similar, and both are similar to the phenotypic factor structure" (p. 235), which reflects both sources of variance. A similar pattern of findings was described by Loehlin and Nichols (1976). Reasons for such findings are not yet clear. Plomin et al. (1990) hazarded one guess: "One possibility is that phenotypic structuring may reflect an underlying physiological structuring that mediates the influence of both genetic and environmental factors" (pp. 235-236). Further studies are needed of the genetic and environmental basis of the covariation between personality and family environment variables.

We have presented the correspondences between the structures of personality attributes and of attributes of family environment simply as an interesting empirical phenomenon, evident in our results, needing explanation. Although we have speculated previously on potential causes for this phenomenon, these data allow only a correlational design that is unable to establish causal bases for the phenomenon.

Increasing scientific understanding of this empirical phenomenon may have important clinical implications. Pincus and Ruiz (1997) found an association between positive memories of caregivers (an important part of family environment) and favorable personality traits. Conversely, maladjustment 
is likely associated with unfavorable family environments; indeed, this is a longstanding assumption of many variants of psychodynamic theory. The results of this study support the existence of homologies between the structures of environment and of personality that undergird these relations. This might suggest a parsimonious principle: Individual attributes and family attributes, or at least the perception of these, tend to correspond.

\section{CONCLUSIONS}

Our study points to a potentially replicable structure of family-environment characteristics. This structure, although evident with a familiar set of nine-item scales from a widely used measure, becomes even more clearly evident when items are rearranged into 22 smaller clusters. Combined with previous literature, these studies have suggested a degree of homology between the structure of characteristics of personality, parents, and family environments as well as a degree of correspondence between ratings of these three kinds of targets. The basis for these correspondences would be an interesting target for future research.

The study of personality would be enhanced by a useful taxonomy of situations (Funder, 2000). However, rather than constituting a taxonomy of situations, a structure of familyenvironment characteristics is really but a subtaxonomy. It is a way of classifying the characteristics in one narrow set of situations: those in which one is interacting with one or more of a circumscribed group of persons (one's family) and typically (although not always) in one particular location (a home). Moreover, situations cannot be defined entirely by who one is with or where one is but also include (at least) the activity that one is currently engaged in, the degree and type of environmental press that one is experiencing, and perhaps even one's own context-altering, subjective state-for example whether one is angry or sad or happy (Saucier et al., in press). There are undoubtedly multiple situation domains. It is possible, however, that dimensions like Active-Passive, Calm-Conflictual, and Structured-Unstructured can be found in multiple situation domains and not only in the fairly narrow one of family environments. This is another possibility that can be considered in future, broader research.

\section{ACKNOWLEDGMENTS}

This work was supported by Grant MH-49227 from the National Institute of Mental Health, U.S. Public Health Service, with recruitment of Samples 1 and 2 supported by Grant R01-DA08417 from the National Institute of Drug Abuse.

\section{REFERENCES}

Beavers, W. R. (1977). Psychotherapy and growth: A family systems perspective. New York: Brunner/Mazel.
Beavers, W. R., Boake, C., \& Salmon, P. G. (1983). Demography correlates and factor structure of the Family Environment Scale. Journal of Clinical Psychology, 39, 95-100.

Cattell, R. B., Coan, R. W., \& Beloff, H. (1958). A re-examination of personality structure in late childhood, and development of the High School Personality Questionnaire. Journal of Experimental Education, 27, 73-88.

Costa, P. T., \& McCrae, R. R. (1992). Revised NEO Personality Inventory (NEO PI-R) and NEO Five-Factor Inventory (NEO-FFI) professional manual. Odessa, FL: Psychological Assessment Resources.

De Raad, B., \& Peabody, D. (2005). Cross-culturally recurrent personality factors: Analyses of three factors. European Journal of Personality, 19, 451-474.

Di Blas, L., \& Forzi, M. (1999). Refining a descriptive structure of personality attributes in the Italian language: The abridged Big Three circumplex structure. Journal of Personality and Social Psychology, 76, 451-481.

Forman, S. G., \& Forman, B. D. (1981). Family environment and its relation to adolescent personality factors. Journal of Personality Assessment, 45, 163-167.

Fowler, P. C. (1981). Maximum likelihood factor structure of the Family Environment Scale. Journal of Clinical Psychology, 37, 160-164.

Fowler, P. C. (1982). Factor structure of the Family Environment Scale: Effects of social desirability. Journal of Clinical Psychology, 38, 285292.

Frederiksen, N. (1972). Toward a taxonomy of situations. American Psychologist, 27, 114-123.

Funder, D. C. (2000). Personality. Annual Review of Psychology, 52, 197221.

Goldberg, L. R. (1972). Parameters of personality inventory construction and utilization: A comparison of prediction strategies and tactics. Multivariate Behavioral Research Monographs, 7, No. 72-2.

Goldberg, L. R. (1990). An alternative "description of personality": The Big-Five factor structure. Journal of Personality and Social Psychology, $59,1216-1229$.

Goodenough, W. H. (1981). Culture, language, and society. Menlo Park, CA: Benjamin/Cummings.

Guadagnoli, E., \& Velicer, W. F. (1988). Relation of sample size to the stability of component patterns. Psychological Bulletin, 103, 265-275.

Halverson, C. F. (1988). Remembering your parents: Reflections on the retrospective method. Journal of Personality, 56, 435-443.

Hathaway, S. R., \& McKinley, J. C. (1943). The Minnesota Multiphasic Personality Inventory. Minneapolis: University of Minnesota Press.

Heck, S. A., \& Pincus, A. L. (2001). Agency and communion in the structure of parental representations. Journal of Personality Assessment, 76, 180184.

Jackson, D. (1974). The Personality Research Form manual. Goshen, NY: Research Psychologists Press.

Kronenberger, W. G., \& Thompson, R. J. (1990). Dimensions of family functioning in families with chronically ill children: Factor analysis of the Family Environment Scale. Journal of Clinical Child Psychology, 19, 380-388.

Kuusinen, J. (1969). Factorial invariance of personality ratings. Scandinavian Journal of Psychology, 10, 33-44.

Lewin, K. (1936). Principles of topological psychology. New York: McGraw-Hill.

Loehlin, J. C., \& Nichols, R. C. (1976). Heredity, environment, and personality: A study of 850 sets of twins. Austin: University of Texas Press.

Magnusson, D. (1981a). Problems in environmental analyses-An introduction. In D. Magnusson (Ed.), Toward a psychology of situations: An interactional perspective (pp. 3-7). Hillsdale, NJ: Lawrence Erlbaum Associates, Inc.

Magnusson, D. (1981b). Wanted: A psychology of situations. In D. Magnusson (Ed.), Toward a psychology of situations: An interactional perspective (pp. 9-32). Hillsdale, NJ: Lawrence Erlbaum Associates, Inc.

McCrae, R. R., \& Costa, P. T. (1988). Recalled parent-child relations and adult personality. Journal of Personality, 56, 417-434. 
McCrae, R. R., \& Costa, P. T. (1989). The structure of interpersonal traits: Wiggins' circumplex and the five-factor model. Journal of Personality and Social Psychology, 56, 586-595.

McCrae, R. R., Costa, P. T., Ostendorf, F., Angleitner, A., Hrebickova, M., Avia, M. D., et al. (2000). Nature over nurture: Temperament, personality and life span development. Journal of Personality and Social Psychology, 78, 173-186.

Moos, R. H. (1974). Family Environment Scale preliminary manual. Palo Alto, CA: Consulting Psychologists Press.

Moos, R. H. (1990). Conceptual and empirical approaches to developing family-based assessment procedures: Resolving the case of the Family Environment Scale. Family Process, 90, 199-208.

Moos, R. H., \& Moos, B. S. (1981). Family Environment Scale manual. Palo Alto, CA: Consulting Psychologists Press.

Moos, R. H., \& Moos, B. S. (1986). Family Environment Scale (2nd ed.). Palo Alto, CA: Consulting Psychologists Press.

Mulaik, S. A. (1964). Are personality factors raters' conceptual factors? Journal of Consulting Psychology, 28, 506-511.

Nunnally, J. C., \& Bernstein, I. H. (1994). Psychometric theory (3rd ed.). New York: McGraw-Hill.

Oliver, J. M., Handal, P. J., Enos, D. M., \& May, M. J. (1988). Factor structure of the Family Environment Scale: Factors based on items and subscales. Educational and Psychological Measurement, 48, 469477.

Oliver, J. M., May, M. J., \& Handal, P. J. (1988). The factor structure of the Family Environment Scale: Factors derived from subscales. Journal of Clinical Psychology, 44, 723-727.

Osgood, C. E. (1966). Dimensionality of the semantic space for communication via facial expressions. Scandinavian Journal of Psychology, 7 , $1-30$.

Osgood, C. E. (1969). On the whys and wherefores of E, P, and A. Journal of Personality and Social Psychology, 12, 194-199.

Osgood, C. E. (1974). Probing subjective culture. Journal of Communication, 24, 21-34, 82-100.

Osgood, C. E., May, W. H., \& Miron, M. S. (1975). Cross-cultural universals of affective meaning. Urbana: University of Illinois Press.

Osgood, C. E., Suci, G. J., \& Tannenbaum, P. H. (1957). The measurement of meaning. Urbana: University of Illinois Press.

Pincus A. L., \& Ruiz, M. A. (1997). Parental representations and dimensions of personality: Empirical relations and assessment implications. Journal of Personality, 68, 436-454.

Plomin, R. (1994). Genetics and experience: The interplay between nature and nurture. Thousand Oaks, CA: Sage.

Plomin, R., Chipuer, H. M., \& Loehlin, J. C. (1990). Behavioral genetics and personality. In L. A. Pervin (Ed.), Handbook of personality: Theory and Research (pp. 225-243). New York: Guilford.

Plomin, R., \& DeFries, J. C. (1983). The Colorado Adoption Project. Child Development, 54, 276-289.

Plomin, R., McClearn, G. E., Pedersen, N. L., Nesselroade, J. R., \& Bergeman, C. S. (1988). Genetic influence on childhood family environment perceived retrospectively from the last half of the life span. Developmental Psychology, 24, 738-745.

Quinlan, D. M., Blatt, S. J., Chevron, E. S., \& Wein, S. J. (1992). The analysis of descriptions of parents: Identification of a more differentiated factor structure. Journal of Personality Assessment, 59, 340-351.

Roosa, M. W., \& Beals, J. (1990). Measurement issues in family assessment: The case of the Family Environment Scale. Family Process, 29, 191198.

Rumpold, V. G., Doering, S., Hofer, S., \& Schussler, G. (2002). A German version of the Measure of Parental Style (MOPS). Zeitschrift fuer Psychosomatische Medizin und Psychotherapie, 48, 5974.

Sanford, K., Bingham, C. R., \& Zucker, R. A. (1999). Validity issues with the Family Environment Scale: Psychometric resolution and research application with alcoholic families. Psychological Assessment, 11, 315325.
Saucier, G. (1997). Effects of variable selection on the factor structure of person-descriptors. Journal of Personality and Social Psychology, 73, 1296-1312.

Saucier, G., Bel-Bahar, T., \& Fernandez, C. (in press). What modifies the expression of personality tendencies? Defining basic domains of situation variables. Journal of Personality.

Saucier, G., \& Goldberg, L. R. (2001). Lexical studies of indigenous personality factors: Premises, products, and prospects. Journal of Personality, $69,847-880$.

Saucier, G., Ostendorf, F., \& Peabody, D. (2001). The non-evaluative circumplex of personality adjectives. Journal of Personality, 69, 537-582.

Schmitt, N. (1996). The uses and abuses of coefficient Alpha. Psychological Assessment, 8, 350-353.

Schwartz, T. (1978). Where is the culture? Personality as the distributive locus of culture. In G. D. Spindler (Ed.), The making of psychological anthropology (pp. 419-441). Berkeley: University of California Press.

Sells, S. B. (1963). An interactionist looks at the environment. American Psychologist, 18, 696-702.

Sines, J. O. (1984). Relations between the Family Environment Scale (FES) and the MMPI. Journal of Personality Assessment, 48, 6-10.

Streiner, D. L. (2003). Starting at the beginning: An introduction to coefficient alpha and internal consistency. Journal of Personality Assessment, 80, 99-103.

Warka, J. L. (1996). Family environment and the development of hardiness. Unpublished master's thesis, Department of Psychology, California State University, San Bernardino.

Widaman, K. F. (1990). Bias in pattern loadings represented by common factor analysis and component analysis. Multivariate Behavioral Research, $25,89-95$.

Wiggins, J. S., Trapnell, P., \& Phillips, N. (1988). Psychometric and geometric characteristics of the Revised Interpersonal Adjective Scales (IAS-R). Multivariate Behavioral Research, 23, 517-530.

\section{APPENDIX}

$(\mathrm{r})=$ reverse-keyed item.

Example Items for the 22 Clusters From the Family Environment Scale.

\section{Solidarity}

There is a feeling of togetherness in our family.

(r) There is very little group spirit in our family.

\section{Supportiveness}

Family members really help and support one another.

Family members really back each other up.

\section{Open Disclosure}

We tell each other about our personal problems.

(r) Family members often keep their feelings to themselves.

\section{Upset Proneness}

It's hard to "blow off steam" at home without upsetting somebody. Someone usually gets upset if you complain in our family.

\section{Cool Temperedness}

Family members rarely become openly angry.

Family members hardly ever lose their tempers.

\section{Combativeness}

We fight a lot in our family.

(r) We really get along with each other. 


\section{Violent Force}

Family members sometimes get so angry they throw things.

Family members sometimes hit each other.

\section{Encouragement}

Family members strongly encourage each other to stand up for their rights. (r) We are not really encouraged to speak up for ourselves in our family.

\section{Achievement Orientation}

We always strive to do things just a little better the next time.

(r) In our family, we don't try that hard to succeed.

\section{Intragroup Competitiveness}

Family members often try to one-up or outdo each other.

Family members are often compared with others as to how well they are doing at work or school.

\section{Education/Culture Orientation}

Family members really like music, art, and literature.

(r) We are not that interested in cultural activities.

\section{Dialogue/Discussions}

We often talk about political and social problems.

(r) We rarely have intellectual discussions.

\section{TV Watching}

Watching TV is more important than reading in our family.

Our main form of entertainment is watching TV or listening to the radio.

\section{Recreational Activities}

We often go to movies, sports events, camping, etc.

(r) Family members are not very involved in recreational activities (outside work or school).

\section{Hobby Orientation}

Everyone in our family has a hobby or two.

Family members sometimes attend courses or take lessons for some hobby or interest (outside of school).

\section{Religious Activities}

Family members attend church, synagogue, or Sunday School fairly often. (r) We don't say prayers in our family.

\section{Fundamentalist Beliefs}

Family members believe that if you sin you will be punished.

(r) We don't believe in heaven or hell.

\section{Neatness}

We are generally very neat and orderly.

Family members make sure their rooms are neat.

\section{Industriousness}

We put a lot of energy into what we do at home.

"Work before play" is the rule in our family.

\section{Strictness}

Family members have strict ideas about what is right and wrong. There is a strong emphasis on following rules in our family.

\section{Autocracy/Overbearingness}

Family members often criticize each other.

(r) Everyone has an equal say in family decisions.

\section{Permissiveness}

We come and go as we want in our family.

We can do whatever we want to in our family.

Gerard Saucier

Department of Psychology

University of Oregon

312 Straub Hall

Eugene, OR 97403-5225

Email: gsaucier@oregon.uoregon.edu

Received April 13, 2006

Revised August 21, 2006 